\title{
Maternal BMI, glucose tolerance, and adverse pregnancy outcomes
}

\begin{abstract}
Alison M. STUEBE, M.D., M.Sc., Mark B. LANDON, M.D., Yinglei LAI, Ph.D., Catherine Y. SPONG, M.D., Marshall W. CARPENTER, M.D., Susan M. RAMIN, M.D., Brian CASEY, M.D., Ronald J. WAPNER, M.D., Michael W. VARNER, M.D., Dwight J. ROUSE, M.D., Anthony SCISCIONE, D.O., Patrick CATALANO, M.D., Margaret HARPER, M.D., M.Sc., George SAADE, M.D., Yoram SOROKIN, M.D., Alan M. PEACEMAN, M.D., and Jorge E. TOLOSA, M.D., MSCE ${ }^{*}$ for the Eunice Kennedy Shriver National Institute of Child Health and Human Development Maternal-Fetal Medicine Units Network, Bethesda, MD

Department of Obstetrics and Gynecology at University of North Carolina at Chapel Hill, Chapel Hill, NC (AMS); The Ohio State University (MBL); Brown University, Providence, RI (MWC); The University of Texas Health Science Center at Houston, Houston, TX (SMR); The University of Texas Southwestern Medical Center, Dallas, TX (BC); Columbia University, New York, NY (RJW); University of Utah, Salt Lake City, UT (MWV); University of Alabama at Birmingham, Birmingham, AL (DJR); Drexel University, Philadelphia, PA (AS); Case Western Reserve University-MetroHealth Medical Center, Cleveland, $\mathrm{OH}(\mathrm{PC})$; Wake Forest University Health Sciences, Winston-Salem, NC (MH); University of Texas Medical Branch, Galveston, TX (GS); Wayne State University, Detroit, MI (YS); Northwestern University, Chicago, IL (MD); Oregon Health \& Science University, Portland, OR (JET); and The George Washington University Biostatistics Center, Washington, DC (YL); and the Eunice Kennedy Shriver National Institute of Child Health and Human Development, Bethesda (CYS)
\end{abstract}

\section{Abstract}

Objectives-To estimate the association of pregravid body mass index (BMI), independent of 3hour oral glucose tolerance test (OGTT) results, with pregnancy outcome.

Study Design-In this secondary analysis of a cohort of women with untreated mild gestational glucose intolerance, defined as $50 \mathrm{~g}$ glucose loading test between 135 and $199 \mathrm{mg} / \mathrm{dL}$ and fasting glucose $<95 \mathrm{mg} / \mathrm{dL}$, we modeled the association between pregravid BMI, OGTT results, and both pregnancy complications and neonatal adiposity.

Results-Among 1250 participants, both pregravid BMI and glucose at hour 3 of the OGTT were associated with increased risk of gestational hypertension. Maternal pregravid BMI was also

\footnotetext{
(C) 2012 Mosby, Inc. All rights reserved.

Correspondence and reprint requests to: Alison M. Stuebe, astuebe @med.unc.edu, Assistant Professor, Division of Maternal-Fetal Medicine, Department of Obstetrics and Gynecology, School of Medicine, University of North Carolina at Chapel Hill, 3010 Old Clinic Building, CB\#7516, Chapel Hill, NC 27599-7516. Work phone: 919-966-1601, Fax: 919-966-6377, Home phone 919-883-4961.

The other members of the Eunice Kennedy Shriver National Institute of Child Health and Human Development (NICHD) MaternalFetal Medicine Units Network are listed in the Appendix.

Preliminary results were presented at the Society for Maternal-Fetal Medicine, San Francisco, CA, February 11, 2011.

Disclosure: None of the authors have a conflict of interest.

Publisher's Disclaimer: This is a PDF file of an unedited manuscript that has been accepted for publication. As a service to our customers we are providing this early version of the manuscript. The manuscript will undergo copyediting, typesetting, and review of the resulting proof before it is published in its final citable form. Please note that during the production process errors may be discovered which could affect the content, and all legal disclaimers that apply to the journal pertain.
} 
positively associated with LGA, and both maternal BMI and fasting glucose were associated with birth-weight z-score and neonatal fat mass.

Conclusions-Among women with untreated mild gestational glucose intolerance, pregravid $\mathrm{BMI}$ is associated with increased gestational hypertension, birth weight and neonatal fat mass, independent of OGTT values.

\section{Keywords}

maternal glycemia; body mass index; obesity; gestational diabetes; GDM

\section{Introduction}

Both maternal obesity ${ }^{1-5}$ and gestational diabetes ${ }^{6}(\mathrm{GDM})$ are associated with an increased risk of adverse pregnancy outcomes. Mothers with these conditions face higher rates of gestational hypertension, preeclampsia, and cesarean delivery ${ }^{7}$. Infants born to these mothers are at increased risk for macrosomia, birth trauma, hypoglycemia and admission to the neonatal intensive care unit. Moreover, these infants are at increased risk for obesity in later life ${ }^{8}$.

Treatment of GDM can reduce complications 9,10 . Obesity is also associated with adverse pregnancy outcome, but the predictive value of maternal pregravid body mass index (BMI) independent of glucose intolerance is not well-defined. If maternal pregravid BMI is associated with adverse outcomes independent of glucose tolerance, then glucose tolerant, obese women may require extra surveillance and/or treatment to mitigate their risk. If, on the other hand, glucose tolerance mediates associations between obesity and adverse outcomes, then obese women with normal glucose tolerance could be considered as low risk and treated as such.

We sought to estimate the association of maternal pregravid BMI, independent of glucose tolerance, with adverse pregnancy outcomes. We therefore measured the independent association of pregnancy outcome with pregravid BMI and parameters of the 3-hour oral glucose tolerance test (OGTT) in a cohort of women with untreated mild gestational glucose intolerance.

\section{Materials and methods}

We performed a secondary analysis of women with untreated mild gestational glucose intolerance. Participants were women randomized to no treatment in the previously reported Eunice Kennedy Shriver National Institute of Child Health and Human Development Maternal-Fetal Medicine Units Network multicenter randomized trial of treatment for mild gestational diabetes (GDM), as well as women in the associated observational cohort, comprised of women with a 50-gram glucose screen $\geq 135 \mathrm{mg} / \mathrm{dL}$ who did not meet criteria for gestational diabetes. ${ }^{9}$ All the women who participated in the study provided written informed consent. The study was approved by the human subjects committee at each participating center.

To be eligible for participation in the primary study, women had to be between 24 weeks 0 days and 30 weeks 6 days gestation and have a 50-gram glucose loading test screen between 135 and $199 \mathrm{mg} / \mathrm{dL}$. Eligible women underwent diagnostic testing with a 100-gram 3-hour OGTT. Women with normal fasting values $(<95 \mathrm{mg} / \mathrm{dL})$ but at least 2 OGTT values exceeding established thresholds ( $1 \mathrm{~h} 180 \mathrm{mg} / \mathrm{dL}, 2 \mathrm{~h} 155 \mathrm{gm} / \mathrm{dL}, 3 \mathrm{~h} 140 \mathrm{mg} / \mathrm{dL}$ ) were randomized to treatment for mild GDM or usual care. In addition, women with normal OGTT results were followed in an observational cohort. Participants in the observational 
cohort were frequency matched at each participating center to the GDM group by body mass index $<$ or $\geq 27 \mathrm{~kg} / \mathrm{m}^{2}$ and race/ethnicity. By design, providers were blinded to OGTT results for women who were randomized to the usual care and observational groups; therefore, clinical care was unrelated to OGTT results. Further details of the methodology of the study have been described elsewhere. ${ }^{9}$ Women were excluded from the primary study if they had any of the following conditions: preexisting diabetes, an abnormal result on a glucose screening test < $24 \mathrm{wks}$, or prior GDM; history of stillbirth, multifetal gestation, asthma, or chronic hypertension; were taking corticosteroids; had a known fetal anomaly, or were likely to have an imminent preterm delivery.

For the current analysis, we excluded women missing data on pregravid BMI $(\mathrm{N}=110)$, leaving 414 women with mild GDM who were randomized to usual care and 836 who were enrolled in the observational cohort. We used maternal pregravid weight and measured height to calculate pregravid BMI in $\mathrm{kg} / \mathrm{m}^{2}$. Maternal weight was abstracted from the prenatal record or obtained by patient report, and height was measured at the time of the 3hour OGTT.

The primary outcome of the parent study was a composite outcome of perinatal mortality, hypoglycemia, hyperbilirubinemia, neonatal hyperinsulinemia, or birth trauma ${ }^{9}$. Gestational hypertension was defined as systolic blood pressure $\geq 140 \mathrm{~mm} \mathrm{Hg}$ or diastolic blood pressure $\geq 90 \mathrm{~mm} \mathrm{Hg}$ or more on two occasions at least 4 hours apart, or one elevated bloodpressure value subsequently treated with medication. Preeclampsia was defined as elevation in blood pressure, as defined for gestational hypertension, with proteinuria ( $2300 \mathrm{mg}$ of protein / 24-hour collection or dipstick $\geq 2+$ if 24 -hour collection not available) or with AST $\geq 70 \mathrm{U} / \mathrm{L}$ or platelet count $<100,000 / \mathrm{L}$. Birth weight $\mathrm{z}$-score was calculated using U.S. singleton birth weight percentiles for gestational age from 1994 to $1996^{11}$. Large for gestational age (LGA) birth weight was defined as $>90 \%$ for gestational age, and small for gestational age (SGA) was defined as birth weight $<10 \%$ for gestational age. To estimate neonatal fat mass ${ }^{12}$, trained research staff measured the infant's length, head and upper midarm circumferences, and flank skin-fold shortly after the infant's birth.

We used Spearman correlation coefficient to measure the association between 3-hour OGTT parameters and maternal pregravid BMI. We then used logistic regression to measure the association between maternal pregravid BMI, OGTT results and the following pregnancy outcomes: composite neonatal outcome, gestational hypertension or preeclampsia, preeclampsia alone, LGA, and SGA. We further used linear regression to model the association between maternal pregravid BMI, OGTT results and birth weight z-score ${ }^{11}$ and neonatal fat mass. All regression models included parity, maternal age and race/ethnicity, maternal pregravid BMI and all four OGTT values. To illustrate the magnitude of differences associated with a range of maternal pregravid BMI, we present predicted probabilities (figure 1) and z-scores and fat mass (figure 2) for each outcome as a function of maternal BMI for a hypothetical multiparous white woman with population mean values for age (27.9 years) and for the oral glucose tolerance test (fasting: 85.3, $1 \mathrm{~h}: 166.5,2 \mathrm{~h}$ : 144.3, 3h: 117.1). The Shieh-O'Brien approximation was used for power calculations ${ }^{13}$. All the statistical analyses were performed using SAS statistical software (SAS Institute, Cary, NC) and R (www.r-project.org).

\section{Results}

Of the 1250 women eligible for inclusion in our analysis, the majority was Hispanic $(54.2 \%)$. The mean maternal age was 27.9 years, and $34 \%$ were nulliparous (Table 1 ). 
Maternal pregravid BMI correlated with fasting 3-hour OGTT results $(\mathrm{r}=0.16, \mathrm{p}<.001)$. BMI was also associated with the 1-hour value on the 3-hour OGTT and inversely associated with 3-hour value. We found correlations among all four 3-hour OGTT parameters (all $\mathrm{p} \leq 0.005$ ), with the strongest correlations among post-load values (Table 2 ).

In logistic regression analyses including both maternal pregravid BMI and 3-hour OGTT parameters, we found that maternal BMI was predictive of outcome independent of OGTT (Table 3). Both maternal BMI and 3-hour OGTT were positively associated with the composite outcome of gestational hypertension or preeclampsia (OR per 5 BMI units: 1.29, 95\%CI 1.10-1.51; OR per $10 \mathrm{mg} / \mathrm{dL}$ 3h glucose: $1.08,95 \%$ CI 1.01-1.17). Maternal pregravid BMI was positively associated with LGA birth weight (OR per 5 BMI units 1.21, 95\%CI 1.03-1.41) and negatively associated with SGA birth weight (OR per 5 BMI units . 77, 95\%CI .60-.96). We found no statistically significant associations between maternal BMI or 3-hour OGTT parameters and preeclampsia or composite neonatal outcome. Maternal BMI was strongly associated with both birth weight z-score and neonatal fat mass. We further found that fasting glucose and 2-hour glucose values were associated with greater birth weight $\mathrm{z}$-score, and fasting glucose values were associated with greater fat mass. Predicted probabilities of gestational hypertension, preeclampsia, LGA and SGA, as well as birth weight $z$-score and fat mass, for a white, multiparous mother aged 27.9 years with mean OGTT results, are presented in Figures 1 and 2.

A post-hoc power analysis was performed to determine the odds ratio for which our sample provided $80 \%$ power with an alpha of 0.05 to detect a statistically significant association between BMI and outcome given the prevalence of preeclampsia (4.4\%) and composite outcome $(34.0 \%)$ in our population. We estimated we had $80 \%$ power to detect a 1.43 -fold OR of preeclampsia and a 1.18-fold OR of composite outcome per 5 BMI units.

\section{Comment}

In a secondary analysis of a cohort of women with 50-gram glucose loading test screen between 135 and $199 \mathrm{mg} / \mathrm{dL}$ and normal fasting glucose, we found that elevated maternal pregravid BMI was associated with hypertensive complications, infant birth weight z-score and fat mass, independent of 3-hour OGTT results. These findings suggest that elevated maternal pregravid BMI is an independent risk factor for adverse birth outcomes and neonatal adiposity, with the caveat that our cohort was limited to women with mild gestational glucose intolerance, and therefore may not be generalizable to women with more severe hyperglycemia.

Our findings confirm and extend earlier work linking maternal BMI and glucose tolerance with pregnancy outcomes. In the HAPO study $(\mathrm{N}=23,316)$, Metzger et al ${ }^{14}$ assessed pregnancy outcomes among women with fasting glucose $<105 \mathrm{mg} / \mathrm{dL}$ and 2-hour, $75 \mathrm{~g}$ OGTT $<200 \mathrm{mg} / \mathrm{dL}$ at 24 to 32 weeks' gestation. They found maternal BMI at study enrollment was directly associated with LGA, primary cesarean section, cord serum cpeptide $>90^{\text {th }}$ percentile, intensive neonatal care, preeclampsia and neonatal adiposity. These associations were attenuated with adjustment for fasting glucose and mean arterial pressure at the time of the OGTT visit, but remained statistically significant for all outcomes except intensive neonatal care. Other authors have similarly found associations between body mass index and adverse perinatal outcome, independent of glucose tolerance ${ }^{15-22}$.

Our finding that 3-hour OGTT values were not associated with adverse outcome reflects our modeling approach. The purpose of our analysis was to determine whether maternal BMI is associated with adverse pregnancy outcomes, independent of glucose tolerance. As expected, we found that the 4 values on the 3-h OGTT are correlated. The inclusion of correlated values in a multiple regression model increases the confidence intervals for each 
variable and increases $\mathrm{p}$ values, reducing the likelihood of detecting an association between each 3-hour OGTT value and pregnancy outcome. This analysis answers a specific clinical question: if two women have the same 3-hour OGTT result, but one has a higher BMI, how much more should a clinician be concerned about the higher BMI patient? This information can assist clinicians in risk-stratifying patients based on pregravid BMI. This is distinct from assessing whether two women with the same BMI, but different values at single value of the OGTT, without considering other OGTT values, have different clinical outcomes. Indeed, other analyses in this cohort, and in other populations, have found that individual fasting and post-load glucose values are associated with increased risk of adverse outcome ${ }^{23,24}$.

Treatment of gestational glucose intolerance reduces adverse outcomes, including macrosomia and gestational hypertensive complications $9,10,25$. The independent association of maternal BMI with these outcomes suggests that glucose tolerant overweight or obese women are similarly at risk for adverse outcomes, and treatment strategies are needed to reduce this risk. Recent randomized controlled trials among overweight women have had mixed results ${ }^{26-28}$. In some cases, intervention participants have improved adherence to dietary guidelines or increased physical activity without a difference in gestational weight gain or metabolic parameters ${ }^{29-31}$. A small pilot study $(\mathrm{N}=50)$ among non-diabetic, obese women found that intensive nutrition consultation reduced gestational weight gain, with a trend toward lower rates of gestational diabetes and pregnancy-induced hypertension ${ }^{32}$. Birth weights were marginally lower in the intervention group. A recent cluster-randomized trial in Finland enrolled 399 women who were euglycemic in early pregnancy but at high risk of GDM. The physical activity, diet and weight gain intervention reduced infant birth weight by $133 \mathrm{~g}$ (95\% CI -231 to -35) and rates of LGA from 19.7 to $12.1 \%(\mathrm{p}=0.04)$. Additional large randomized trials, some of which are underway ${ }^{33}$, are needed to confirm or refute the utility of lifestyle intervention to improve outcomes among glucose-tolerant overweight or obese pregnant women and their infants.

Strengths of our secondary analysis include standardized, prospective assessment of glucose tolerance, maternal height, and obstetrical outcomes in the parent trial. The multicenter design of the parent trial included women from 14 centers in the United States, increasing generalizability. Nevertheless, our findings must be interpreted in the context of the study design. This was a secondary analysis of participants in a clinical trial, and enrollment was limited to women with moderately elevated glucose loading test results but a fasting glucose $<95 \mathrm{mg} / \mathrm{dL}$. Our results are therefore only generalizable to women with mild glucose intolerance. Because the study enrollment criteria constrained glucose parameters, but not body mass index, we were more likely to detect an association with maternal BMI than with OGTT parameters. This sample bias may contribute to our findings that 3-hour OGTT parameters did not independently predict pregnancy outcomes. Further, observational cohort participants were frequency-matched to GDM participants by maternal obesity status, defined as BMI $<27$ vs. $\geq 27 \mathrm{~kg} / \mathrm{m}^{2}$. These selection criteria may further affect generalizability of our results. In addition, pregravid BMI was obtained from chart abstraction or maternal recall, and thus may be subject to misclassification. If heavier women were likely to underreport pregravid $\mathrm{BMI}^{34}$, this misclassification would bias our results toward the null. Nevertheless, we found statistically significant associations between pregravid BMI and both hypertensive complications and extremes of birth weight. Further, the low prevalence of preeclampsia in our cohort reduced our power to detect associations between BMI and preeclampsia in our cohort. However, point estimates obtained in our sample suggest that BMI is more strongly associated with gestational hypertension than with preeclampsia. Finally, we measured associations between pregravid BMI and seven clinical outcomes, but we did not adjust for multiple comparisons. The rationale for this approach was that our goal was to estimate the strength of associations between maternal body mass index and obstetrical complications, independent of maternal glycemia, rather than to test 
whether a specific a priori hypothesis was true or false. Nevertheless, it is possible that some of the observed associations were due to chance.

In conclusion, we found a direct association between maternal pregravid BMI and both gestational hypertension and neonatal adiposity, independent of oral glucose tolerance test parameters. Clinical trials are needed to determine whether dietary and lifestyle interventions among women with elevated pregravid BMI can reduce gestational hypertension and mitigate the intergenerational transmission of obesity.

\title{
Acknowledgments
}

\begin{abstract}
The project described was supported by grants from the Eunice Kennedy Shriver National Institute of Child Health and Human Development (NICHD) [HD27915, HD34116, HD40485, HD34208, HD27869, HD40500, HD40560, HD34136, HD40544, HD27860, HD40545, HD53097, HD21410, HD27917, HD40512, HD53118, HD36801], General Clinical Research Centers Grant [M01-RR00034] and the National Center for Research Resources [UL1RR024989, M01-RR00080, UL1-RR025764, C06-RR11234]. Dr. Stuebe receives support from the Women's Reproductive Health Research Career Development Center at University of North Carolina [5K12HD050113-04]. This work does not necessarily represent the official views of the NICHD or NIH.

The author thanks the following MFMU Network members who participated in protocol development and coordination between clinical research centers (Francee Johnson, R.N., M.S.N. and Jo-Ann Tillinghast, R.N., M.S.N.), protocol/data management and statistical analysis (Elizabeth Thom, Ph.D.), and protocol development and oversight (John M. Thorp, Jr., M.D.).
\end{abstract}

\section{Appendix}

In addition to the authors, other members of the Eunice Kennedy Shriver National Institute of Child Health and Human Development Maternal-Fetal Medicine Units Network are as follows: University of North Carolina at Chapel Hill - J. Thorp, K. Dorman, S. Brody, S. Timlin, J. Bernhardt University of Texas Southwestern Medical Center - K. Leveno, L. Moseley, J. Gold, D. Bradford, L. Fay, M. Garcia, F. Capellan

Columbia University - M. Miodovnik, F. Malone, S. Bousleiman, H. Husami, V. Carmona, N. Fredericks, E. Gantioqui, B. Greenspan, M. Williams

University of Utah - K. Anderson (University of Utah Health Sciences Center), P. Ashby (University of Utah Health Sciences Center), S. McAllister (University of Utah Health Sciences Center), S. Quinn (LDS Hospital), A. Guzman (McKay-Dee Hospital), F. Castinella (LDS Hospital), J. Steiner (McKay-Dee Hospital), J. Parker (Utah Valley Regional Medical Center)

University of Alabama at Birmingham - J. Sheppard, J. Tisdale, A. Northen, W. Andrews Brown University - D. Catlow, D. Allard, M. Seebeck, J. Tillinghast

The Ohio State University — J. Iams, F. Johnson, C. Latimer, E. Weinandy, B. Maselli

Drexel University - M. Hoffman, E. Guzman, M. Talucci, T. Grossman, C. Perez, L. Zeghibe, P. Tabangin

Case Western Reserve University-MetroHealth Medical Center - B. Mercer, B. Stetzer, C. Milluzzi, W. Dalton, S. Pichette

Wake Forest University Health Sciences - M. Swain, P. Meis, J. White

The University of Texas Health Science Center at Houston - L. Gilstrap, K. Cannon, J. Martinez, D. Dusek 
University of Texas Medical Branch - J. Moss, J. Brandon, A. Jackson, G. Hankins, D. Sharp

University of Pittsburgh - S. Caritis, M. Bickus, H. Birkland, M. Cotroneo, N. Cuddy

Wayne State University - G. Norman, P. Lockhart, S. Blackwell, L. Quast

Northwestern University - P. Simon, G. Mallett

Oregon Health \& Science University - L. Davis, E. Lairson, C. Cromett, C. Naze, M. Blaser

The George Washington University Biostatistics Center - E. Thom, L. Mele, J. Zachary, B. Getachew, C. Cobb, L. Leuchtenburg, S. Gilbert

Eunice Kennedy Shriver National Institute of Child Health and Human Development - S. Tolivaisa, K. Howell

MFMU Network Steering Committee Chair (University of Texas Medical Branch, Galveston, $T X)$ - G.D. Anderson, M.D.

\section{References}

1. Bhattacharya S, Campbell DM, Liston WA. Effect of Body Mass Index on pregnancy outcomes in nulliparous women delivering singleton babies. BMC Public Health. 2007; 7:168. [PubMed: 17650297]

2. Callaway LK, Prins JB, Chang AM, McIntyre HD. The prevalence and impact of overweight and obesity in an Australian obstetric population. Med J Aust. 2006; 184:56-9. [PubMed: 16411868]

3. Driul L, Cacciaguerra G, Citossi A, Martina MD, Peressini L, Marchesoni D. Prepregnancy body mass index and adverse pregnancy outcomes. Arch Gynecol Obstet. 2008; 278:23-6. [PubMed: 18071728]

4. Khashan AS, Kenny LC. The effects of maternal body mass index on pregnancy outcome. Eur J Epidemiol. 2009; 24:697-705. [PubMed: 19653107]

5. Sebire NJ, Jolly M, Harris JP, et al. Maternal obesity and pregnancy outcome: a study of 287,213 pregnancies in London. Int J Obes Relat Metab Disord. 2001; 25:1175-82. [PubMed: 11477502]

6. American College of Obstetricians and Gynecologist Committee on Practice Guidelines-Obstetrics. ACOG Practice Bulletin Number 30: Gestational Diabetes. Obstet Gynecol. 2001; 98:525-38. [PubMed: 11547793]

7. Nelson SM, Matthews P, Poston L. Maternal metabolism and obesity: modifiable determinants of pregnancy outcome. Hum Reprod Update. 2010; 16:255-75. [PubMed: 19966268]

8. Hillier TA, Pedula KL, Schmidt MM, Mullen JA, Charles MA, Pettitt DJ. Childhood obesity and metabolic imprinting: the ongoing effects of maternal hyperglycemia. Diabetes Care. 2007; 30:2287-92. [PubMed: 17519427]

9. Landon MB, Spong CY, Thom E, et al. A multicenter, randomized trial of treatment for mild gestational diabetes. N Engl J Med. 2009; 361:1339-48. [PubMed: 19797280]

10. Crowther CA, Hiller JE, Moss JR, McPhee AJ, Jeffries WS, Robinson JS. Effect of treatment of gestational diabetes mellitus on pregnancy outcomes. N Engl J Med. 2005; 352:2477-86. [PubMed: 15951574]

11. Alexander GR, Kogan MD, Himes JH. 1994-1996 U.S. singleton birth weight percentiles for gestational age by race, Hispanic origin, and gender. Matern Child Health J. 1999; 3:225-31. [PubMed: 10791363]

12. Catalano PM, Thomas AJ, Avallone DA, Amini SB. Anthropometric estimation of neonatal body composition. Am J Obstet Gynecol. 1995; 173:1176-81. [PubMed: 7485315]

13. Shieh G. A Comparison of Two Approaches for Power and Sample Size Calculations in Logistic Regression Models. Communications in Statistics - Simulation. 2000; 29:763-91. 
14. HAPO Study Cooperative Research Group. Hyperglycaemia and Adverse Pregnancy Outcome (HAPO) Study: associations with maternal body mass index. Bjog. 2010; 117:575-84. [PubMed: 20089115]

15. Bo S, Menato G, Signorile A, et al. Obesity or diabetes: what is worse for the mother and for the baby? Diabetes Metab. 2003; 29:175-8. [PubMed: 12746640]

16. Ehrenberg HM, Mercer BM, Catalano PM. The influence of obesity and diabetes on the prevalence of macrosomia. Am J Obstet Gynecol. 2004; 191:964-8. [PubMed: 15467573]

17. Jensen DM, Damm P, Sorensen B, et al. Pregnancy outcome and prepregnancy body mass index in 2459 glucose-tolerant Danish women. Am J Obstet Gynecol. 2003; 189:239-44. [PubMed: 12861169]

18. Lapolla A, Bonomo M, Dalfra MG, et al. Prepregnancy BMI influences maternal and fetal outcomes in women with isolated gestational hyperglycaemia: a multicentre study. Diabetes Metab. 2010; 36:265-70. [PubMed: 20598607]

19. Owens LA, O'Sullivan EP, Kirwan B, Avalos G, Gaffney G, Dunne F. ATLANTIC DIP: the impact of obesity on pregnancy outcome in glucose-tolerant women. Diabetes Care. 2010; 33:5779. [PubMed: 20067952]

20. Ricart W, Lopez J, Mozas J, et al. Body mass index has a greater impact on pregnancy outcomes than gestational hyperglycaemia. Diabetologia. 2005; 48:1736-42. [PubMed: 16052327]

21. Schaefer-Graf UM, Heuer R, Kilavuz O, Pandura A, Henrich W, Vetter K. Maternal obesity not maternal glucose values correlates best with high rates of fetal macrosomia in pregnancies complicated by gestational diabetes. J Perinat Med. 2002; 30:313-21. [PubMed: 12235720]

22. Yogev Y, Langer O, Xenakis EM, Rosenn B. The association between glucose challenge test, obesity and pregnancy outcome in 6390 non-diabetic women. J Matern Fetal Neonatal Med. 2005; 17:29-34. [PubMed: 15804783]

23. Landon MB, Mele L, Spong CY, et al. The Relationship Between Maternal Glycemia and Perinatal Oucome. Obstet Gynecol. 2011; 117:218-24.10.1097/AOG.0b013e318203ebe0 [PubMed: 21309194]

24. The Hapo Study Cooperative Research Group. Hyperglycemia and Adverse Pregnancy Outcomes. N Engl J Med. 2008; 358:1991-2002. [PubMed: 18463375]

25. Horvath K, Koch K, Jeitler K, et al. Effects of treatment in women with gestational diabetes mellitus: systematic review and meta-analysis. Bmj. 2010; 340:c1395. [PubMed: 20360215]

26. Dodd JM, Crowther CA, Robinson JS. Dietary and lifestyle interventions to limit weight gain during pregnancy for obese or overweight women: a systematic review. Acta Obstet Gynecol Scand. 2008; 87:702-6. [PubMed: 18607830]

27. Dodd JM, Grivell RM, Crowther CA, Robinson JS. Antenatal interventions for overweight or obese pregnant women: a systematic review of randomised trials. Bjog. 2010; 117:1316-26. [PubMed: 20353459]

28. Ronnberg AK, Nilsson K. Interventions during pregnancy to reduce excessive gestational weight gain: a systematic review assessing current clinical evidence using the Grading of Recommendations, Assessment, Development and Evaluation (GRADE) system. Bjog. 2010; 117:1327-34. [PubMed: 20840691]

29. Kinnunen TI, Pasanen M, Aittasalo M, et al. Preventing excessive weight gain during pregnancy a controlled trial in primary health care. Eur J Clin Nutr. 2007; 61:884-91. [PubMed: 17228348]

30. Callaway LK, Colditz PB, Byrne NM, et al. Prevention of gestational diabetes: feasibility issues for an exercise intervention in obese pregnant women. Diabetes Care. 2010; 33:1457-9. [PubMed: 20357374]

31. Guelinckx I, Devlieger R, Mullie P, Vansant G. Effect of lifestyle intervention on dietary habits, physical activity, and gestational weight gain in obese pregnant women: a randomized controlled trial. Am J Clin Nutr. 2010; 91:373-80. [PubMed: 19955397]

32. Wolff S, Legarth J, Vangsgaard K, Toubro S, Astrup A. A randomized trial of the effects of dietary counseling on gestational weight gain and glucose metabolism in obese pregnant women. Int $\mathbf{J}$ Obes (Lond). 2008; 32:495-501. [PubMed: 18227847] 
33. Dodd JM, Turnbull DA, McPhee AJ, Wittert G, Crowther CA, Robinson JS. Limiting weight gain in overweight and obese women during pregnancy to improve health outcomes: the LIMIT randomised controlled trial. BMC Pregnancy Childbirth. 2011; 11:79. [PubMed: 22026403]

34. Krul AJ, Daanen HA, Choi H. Self-reported and measured weight, height and body mass index (BMI) in Italy, the Netherlands and North America. Eur J Public Health. 2011; 21:414-9. [PubMed: 20089678] 

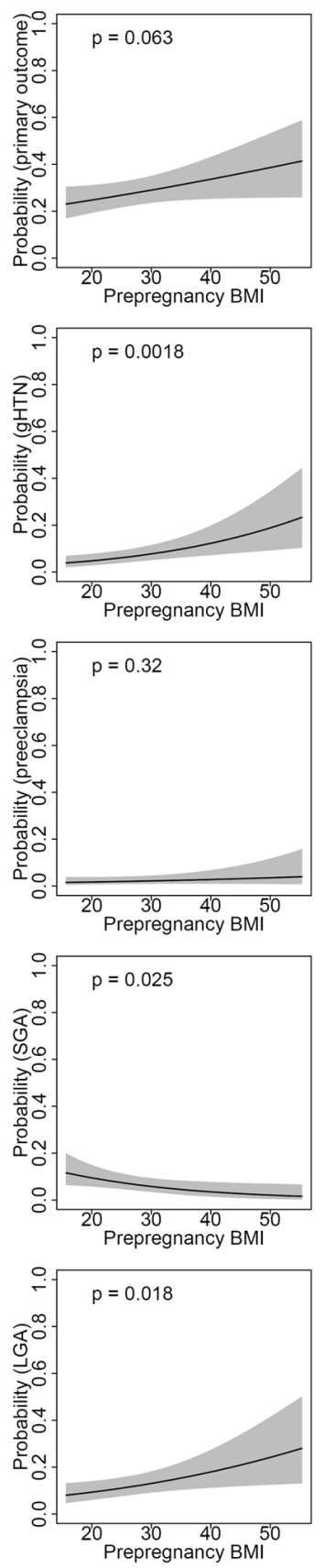

Figure 1.

Predicted probability* of primary outcome, gestational hypertension, preeclampsia, SGA and LGA, by prepregnancy maternal BMI.

Predicted probabilities of pregnancy outcome presented for a hypothetical multiparous white women with mean values for age (27.9) and OGTT values (fasting: 85.3, 1h: 166.5, 2h: 144.3, 3h: 117.1). Models include parity, maternal age, race/ethnicity, maternal pregravid BMI and all four OGTT values. 

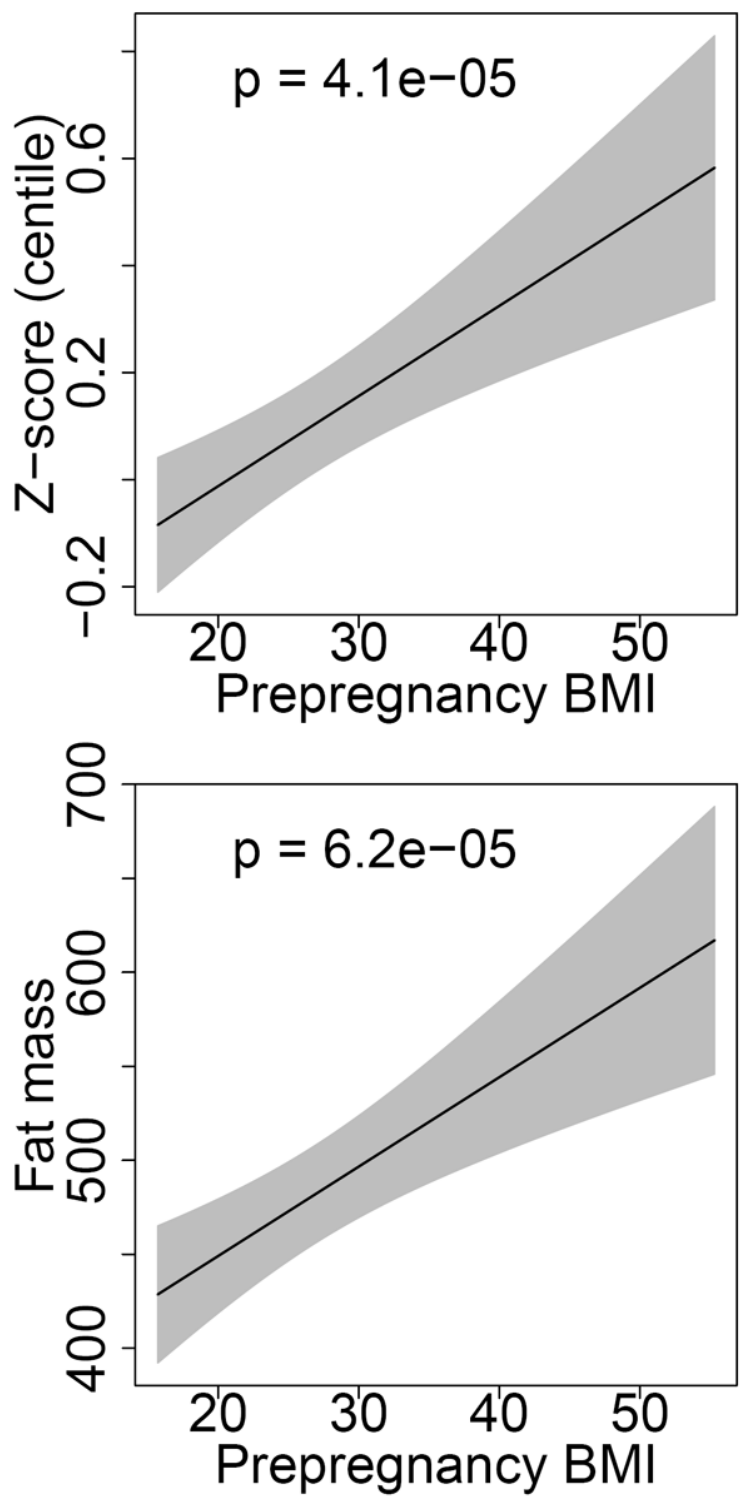

Figure 2.

Predicted birth weight z-score and fat mass by prepregnancy maternal BMI.

Predicted birth weight z-score and neonatal fat mass presented for a hypothetical multiparous white women with mean values for age (27.9) and OGTT values (fasting: 85.3, 1h: 166.5, 2h: 144.3, 3h: 117.1). Models include parity, maternal age, race/ethnicity, maternal pregravid BMI and all four OGTT values. 
Table 1

Characteristics of the study population $(\mathrm{N}=1250)$

\begin{tabular}{|l|l|}
\hline & $\%(\mathbf{N})$ \\
\hline Race/Ethnicity & \\
\hline Black & $12.9(161)$ \\
\hline White & $28.5(356)$ \\
\hline Hispanic & $54.2(678)$ \\
\hline Other & $4.4(55)$ \\
\hline Parity & \\
\hline Nulliparous & $423(33.8)$ \\
\hline Multiparous & $827(66.2)$ \\
\hline BMI & \\
\hline Normal BMI $\left(<25 \mathrm{~kg} / \mathrm{m}^{2}\right)$ & $546(43.7)$ \\
\hline Overweight BMI $\left(25-<30 \mathrm{~kg} / \mathrm{m}^{2}\right)$ & $410(32.8)$ \\
\hline Obese BMI ( $\left.230 \mathrm{~kg} / \mathrm{m}^{2}\right)$ & $294(23.5)$ \\
\hline & Mean (SD) \\
\hline BMI (kg/m2) & $26.6(5.6)$ \\
\hline Age, mean $(\mathrm{SD})$ & $27.9(5.6)$ \\
\hline Fasting glucose & $85.3(5.9)$ \\
\hline $1 \mathrm{~h}$ glucose, $\mathrm{mg} / \mathrm{dL}$ & $166.5(30.2)$ \\
\hline 2h glucose & $144.3(29.5)$ \\
\hline $3 \mathrm{~h}$ glucose & $117.1(28.5)$ \\
\hline
\end{tabular}




\section{Table 2}

Correlations * among maternal pregravid BMI and parameters of the 3-hour 100 gram oral glucose tolerance test.

\begin{tabular}{lrrrr}
\hline \multicolumn{5}{c}{ Oral glucose tolerance test (OGTT) } \\
& Fasting & 1-hour & 2-hour & 3-hour \\
Pregravid BMI & 0.159 & 0.068 & 0.011 & -0.086 \\
& $<.0001$ & 0.016 & 0.687 & 0.002 \\
3-hour OGTT & 0.089 & 0.293 & 0.439 & \\
& 0.002 & $<.0001$ & $<.0001$ & \\
2-hour OGTT & 0.153 & 0.668 & & \\
& $<.0001$ & $<.0001$ & & \\
1-hour OGTT & 0.233 & & & \\
& $<.0001$ & & & \\
\hline
\end{tabular}

* Spearman r, p-value 


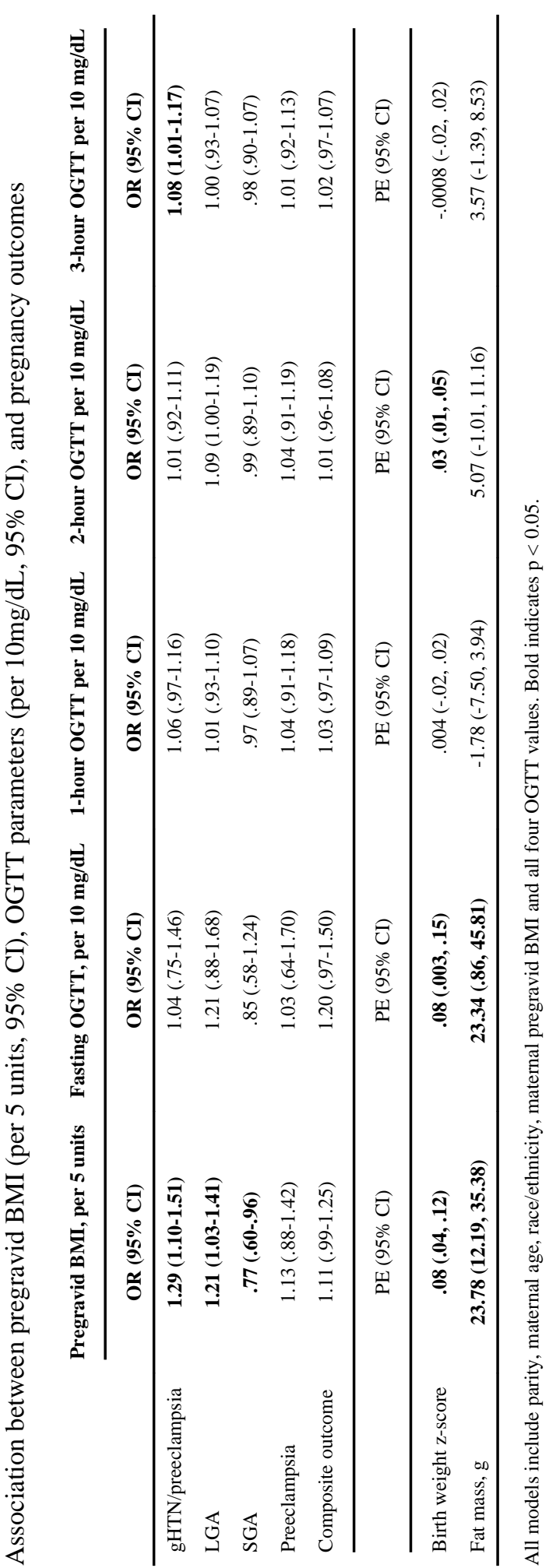

